Geopolítica(s) Revista de estudios sobre espacio y poder ISSN: 2172-3958

https://dx.doi.org/10.5209/geop.70702

\title{
El legado de la geografía tropical: la escuela de Burdeos
}

\author{
Hugo Capellà Miternique ${ }^{1}$
}

Recibido: 24 de abril de 2020 / Aceptado: 4 de octubre de 2020

Resumen. Aún lejos de la sombra de los trópicos, la geografía en Europa encontró bajo los estudios tropicales el alero para el desarrollo de una rica visión regionalista de la que luego la academia se encargaría de renegar por motivos políticos. El proceso de descolonización arrinconó una rica visión regionalista, a pesar de estar en el origen de muchos de los departamentos de geografía. Los estudios tropicales, al margen de los discursos de poder, significaron una mirada plural en el mundo que, tras silenciarse, empieza a retomar fuelle. El presente artículo indaga un caso del legado académico colonial a partir del análisis de documentos y entrevistas a discípulos de la geografía tropical de la Escuela de Burdeos. Tras haber sido arrinconado, se empieza a reconsiderar la diversidad de su bagaje desde una mirada postcolonial.

Palabras clave: geografía tropical; geografía regional; postcolonialismo; revisionismo; Burdeos.

\section{[en] The Legacy of Tropical Geography: The Bordeaux School}

\begin{abstract}
Still far from the shadow of the tropics, geography in Europe found under the tropical studies the roof for the development of a rich regionalist vision that the academy would deny later for political reasons. The decolonization process cornered a rich regionalist vision, despite being in the origin of many geography departments. Tropical studies, leaving aside the discourses of power, signified a plural look at the world that after becoming silent begins to regain its strength. This article investigates a case of the colonial academic legacy from document analysis and the results of interviews with disciples of tropical geography from the Bordeaux School. After being cornered, the diversity of its background is beginning to be reconsidered from a postcolonial perspective.
\end{abstract}

Keywords: tropical geography; regional geography; postcolonialism; revisionism; Bordeaux.

\section{[pt] O legado da geografia tropical: a escola de Bordeaux}

Resumo. Ainda longe da sombra dos trópicos, a geografia europeia encontrou nos estudos tropicais o beiral para o desenvolvimento de uma rica visão regionalista que a academia mais tarde se encarregaria de negar por razões políticas. O processo de descolonização encurralou uma rica visão regionalista, apesar de estar na origem de muitos departamentos de geografia. Os estudos tropicais, à margem dos discursos de poder, significaram um olhar plural para o mundo que, após ser silenciado, começa a recuperar sua força. Este artigo investiga um caso do legado acadêmico colonial a partir da análise de documentos e entrevistas com discípulos da geografia tropical da Escola de Bordeaux. Depois de

1 Department de Geografia, Universitat de les Illes Balears, Palma de Mallorca (España).

E-mail: hugo.capella@uib.eu 
encurralado, a diversidade de sua formação começou a ser reconsiderada a partir de uma perspetiva pós-colonial.

Palavras-chave: geografia tropical; geografia regional; pós-colonialismo; revisionismo; Bordeaux.

Sumario. Introducción: el legado de la geografía tropical. 1. El peso de la herencia geográfica colonial. 1.1. El regionalismo y la academia. 1.2. La geografía tropical de Burdeos. 2. La revisión regional contemporánea. 2.1. El rescate de la experiencia. 2.2. La revalorización del discurso. 3. Conclusión: la mirada tropical. 3.1. La revisión del referente. 3.2. ¿Los trópicos descoloniales?. Agradecimientos. Referencias.

Cómo citar: Capellà Miternique, H. (2020). El legado de la geografía tropical: la escuela de Burdeos. Geopolítica(s). Revista de estudios sobre espacio y poder, 11(2), 345-363.

\section{Introducción: el legado de la geografía tropical}

La relación del legado colonial respecto a los principios universales sobre los cuales se vertebran sus sociedades herederas ha sido un tema de difícil encaje en la geografía (Luna-Nemecio, 2019). El surgimiento del pensamiento crítico sobre el mundo postcolonial deriva por un lado de los movimientos políticos de independencia de antiguas metrópolis (Balandier, 1973), pero por otra parte también desde la valorización y recuperación de unos referentes propios que hasta la fecha no tenían voz propia en las nuevas soberanías recuperadas. También desde las antiguas metrópolis coloniales (Zusman, 2013) se ha desarrollado un pensamiento crítico de forma global (Grosfoguel, 2006).

En el caso de la antigua colonia, la mirada crítica postcolonial permite el reconocimiento propio liberador pese a un pasado complejo de dependencia (de ultramar) a veces difícil de asumir o incluso aún pendiente. Por el contrario, en el caso de la antigua metrópoli, la mirada postcolonial se convierte en la difícil revisión de tener que asumir la responsabilidad contemporánea de un error, así como de enmendar sus consecuencias, tales como por ejemplo, el reconocimiento de los derechos de los antiguos súbditos como nuevos inmigrantes (Estermann, 2014).

El legado colonial en las metrópolis paso al olvido, en primer lugar, por ser una pérdida vergonzosa desde las lógicas nacionales aún coloniales (sirva de ejemplo la pérdida de Cuba para España, o la de Argelia para Francia), así como, en segundo lugar, por entenderse como una etapa de dominación superada ante la mirada fijada en un nuevo marco de modernidad de espaldas al pasado.

Tanto desde el punto de vista político, como social y académico, los focos de interés fueron basculando hacia nuevos temas dejando en el olvido ese complejo y comprometedor pasado. La mirada sobre el mundo colonial pasó a convertirse en un tema políticamente incorrecto y el hablar de ello significaba vincularse con la ideología que lo avaló. Con el auge del pensamiento postcolonial postmoderno (Young, 2016), surgido sobre todo dentro del ámbito anglosajón y más concretamente incentivado por el apoyo institucional del estudio y reivindicación de las minorías en EE UU - no sólo raciales sino también de género- (Tomasi, 1995) pero también desde la revisión en ciertos ámbitos académicos, como la literatura anglo-caribeña (Edmondson, 1999), llegaron indirectamente a las metrópolis de la mano de los inmigrantes o antiguos ciudadanos que diversificaban sus ciudades (Nash, 2005). 
La revalorización social de esas minorías gracias a la consideración de la diversidad (Bailón, 2015) llevó también a la revisión del legado colonial, no como apología de un modelo sino como replanteo de las lógicas supremacistas de esos discursos. En ese contexto, los debates postcoloniales permiten un proceso revisionista (Sidaway, 2016) de un legado europeo que ya se encuentra lo suficientemente lejos temporalmente como para poder ser abordado con la distancia necesaria, puesto que muchos de sus artífices y beneficiarios directos ya han muerto.

En este contexto, la revisión postcolonial de la disciplina de la geografía es muy compleja por la estrecha relación que tuvo con el auge del modelo colonial, sólo comparable con el legado etnográfico para la antropología y en algunos planteamientos filosóficos (González, 2003). Dentro de la disciplina geográfica, el caso de la geografía regional francesa junto, seguramente, con el caso de la Geopolitik alemana (López Trigal, 2017) sean los dos legados de principios del siglo XX más complejos para ser abordados, por sus estrechas vinculaciones con ciertos modelos políticos, francés y alemán respectivamente (Santini, 2018b), de compleja justificación.

En ambos casos, los geógrafos optaron por obviar esas corrientes, al menos en los ámbitos más académicos, para desvincularse y mirar hacia el frente, en una especie de fuga hacia delante amparada en la ciencia, en este caso vinculada a las corrientes neopositivistas de la segunda mitad del siglo XX (Lindón, Hiernaux y Bertrand, 2006). En el caso específico de la geografía francesa, una de sus principales escuelas surgió en Burdeos, uno de los puertos coloniales históricos por excelencia, que servía de conexión del Imperio francés con ultramar y los trópicos. El desarrollo del Departamento de Geografía y de las temáticas de estudio asociadas a él sirvió para reforzar un mundo académico y científico que quedaría ulteriormente silenciado, al no saber desvincularse de su apoyo al desarrollo del modelo colonial. Tras un largo silencio, se ha iniciado un proceso de revisión del legado regional dentro de la geografía desde el punto de vista académico - ya en antecedentes como Wagner y Mikesell (1962) y Berdoulay $(1981,1988)$ - , pero la revisión postcolonial del legado de la geografía tropical de Burdeos sigue pendiente.

El presente artículo plantea la revisión crítica de los discursos de la Escuela tropical de Burdeos analizando, por un lado, la literatura del grupo y, por otro, los resultados de entrevistas a algunos de los discípulos de esta escuela (Joan Vilà Valentí, Pedro Ilabaca y Paul Claval) para desvincular el contexto colonial del discurso complejo y plural del particularismo tropical, muy en sintonía en nuestras sociedades multiculturales contemporáneas. Este ejemplo permite entender la relevancia de la revisión crítica postcolonial del legado académico, sobre todo como parte del proceso de reconocimiento colonial desde la complejidad (Kapoor, 2008).

\section{El peso de la herencia geográfica colonial}

El legado colonial, largamente vinculado con el desarrollo mismo del crecimiento académico y financiero de la disciplina geográfica, ha dado paso a una revisión crítica constructiva que permite desvincular el contexto histórico de los contenidos académicos. Su olvido, aunque fuera dentro de una lógica aséptica de alejamiento ideológico, no hizo más que silenciar toda una etapa histórica sin la posibilidad de una revisión constructiva que permitiera salir de ciertos maniqueísmos simplistas, bajo la etiqueta "colonial", aunque fuera desde una discriminación positiva. 
El legado de la geografía ideográfica, desde su vertiente enciclopédica y descriptiva, está estrechamente relacionada con las expediciones y descubrimientos coloniales, y vinculada con los intereses políticos de los financiadores (Talledos, 2014). La geografía académica moderna intentó ulteriormente desvincularse de este legado, poco científico. Pero buena parte de sus conocimientos - y la disciplina mismaemanan de ese pasado, sin que por ello puedan reducirse a planteamientos colonialistas o eurocéntricos, puesto que el interés de conocimientos geográficos se ha desarrollado en todas las culturas, como una forma de conocimiento particular que apor$\tan \operatorname{los}$ territorios.

\subsection{El regionalismo y la academia}

La geografía descriptiva, inductiva y de raíz ideográfica se tradujo en el mundo académico con el desarrollo de la escuela regional francesa (Claval y Sanguin, 1996) de corte corológico y centrada en explicar la diversidad y complejidad de las particularidades de las regiones de todo el mundo o "genres de vie" (Vidal de La Blache, 1908, 2008; Gallois, 1909). Su relación con la larga tradición geográfica vernácula y el hecho de no renegar del rico legado de las expediciones y el trabajo de campo la hizo ser devaluada dentro del mundo académico, al considerar la tarea descriptiva y la finalidad particularista —o excepcionalista - poco apta para la construcción de un conocimiento científico basado en leyes universales (Zusman, 2011).

La geografía ulterior neopositivista se alejaría de ese pasado para encontrar un lugar propio con el resto de disciplinas científicas (nomotéticas). En ese contexto el bagaje de la escuela regional francesa pudo seguir refugiándose en muchos de los centros de investigación especializados en regiones del mundo (centros de estudios africanos, asiáticos, americanos, oceánicos), financiados por Estados con intereses colonialistas (Unwin, 1995).

El ulterior proceso de descolonización (a partir de los 50s) incidió en la desaparición (por falta de inversión por parte de los Estados) o reconversión (por ejemplo, en el caso de Burdeos, con la creación del Centro de Estudios Tropicales) de los centros de investigación. Esa línea geográfica quedaría estigmatizada tanto desde el punto de vista académico como político, cayendo en un olvido generalizado (Lindón, Hiernaux y Bertrand, 2006).

El cambio generacional e ideológico aparcó la disciplina regional que había estado en muchos casos al origen de la expansión de la disciplina en muchas universidades de muchos países. En el contexto de habla hispana, la influencia regional estuvo al origen de la apertura de muchos departamentos de geografía, en España pero también en América Latina, que luego derivarían hacia tendencias más de geografía humana con el auge de los estudios agrarios en Argentina (como en Bahía Blanca o en Mendoza) y España (Barcelona o Madrid) o de disciplinas más técnicas en el caso chileno (Santiago y Concepción), por citar algunos ejemplos. El impacto de los conocimientos de la geografía regional no sólo se dio en Francia sino que se expandió por todo el mundo gracias a la especialización de geógrafos de todo el mundo en Francia, así como gracias a la labor de difusión de geógrafos regionales franceses por todo el mundo, como por ejemplo en el mundo mediterráneo, árabe (ver, por ejemplo, Gsell, 1997) América Latina (ver, por ejemplo, Laugénie, 1971), África, Indochina (ver, por ejemplo, Gourou, 1965) o Polinesia. El papel de profesores como De Civit, Capitanelli (ver, por ejemplo, Capitanelli y Zamorano, 1972) y Zamorano 
(ver, por ejemplo, Zamorano, 1959) en la Universidad de Cuyo en Argentina, Joan Vilà Valentí en La Universidad de Barcelona, Pedro Ilabaca en la Universidad de Concepción (ver, por ejemplo, Ilabaca, 1993), por citar algunos casos, fueron claves para el despegue de la disciplina geográfica en numerosos países. Así por ejemplo, podemos nombrar cómo los trabajos de Jean Borde (ver, por ejemplo, Borde, 1966) o de Claude Laugénie (ver, por ejemplo, Laugénie, 1971) fueron un aporte valioso no sólo para un mejor conocimiento geográfico de Chile sino que permitieron la llegada de un conocimiento geográfico y técnico que serviría para el arranque de la geografía nacional y dejaría a su vez escuela (Borde, 1980), en geógrafos discípulos chilenos, como la profesora María Mardones. En el caso de la geografía y ciencias sociales catalanas, la influencia francesa fue primordial, figuras como Pierre Vilar (ver, por ejemplo, Vilar, 1962) o Fernand Braudel (ver, por ejemplo, Braudel, 1976), dejaron una profunda huella, en autores como, Joan Vilà Valentí (ver, por ejemplo, Vilà Valentí, 1983) o J. Vicens Vives (ver, por ejemplo, Vicens Vives, 1961) que influenciarían en la formación de nuevos geógrafos, incluso aunque luego fuera para rebelarse, como Horacio Capel o Roser Majoral. La ruptura generacional y epistemológica de la geografía humana respecto a los planteamientos regionales esconde de hecho una continuidad que se evidencia en el origen regional de la geografia humana en la obra de Jean Brunhes (ver, por ejemplo, Brunhes, 1920).

La importancia y rápida expansión del conocimiento regional se debió en gran parte, justamente por su carácter humanista - como en la obra de Mâle (Russo, 2004) - y profundamente cercano a la población, como instrumento pedagógico en la construcción de los discursos e identidades nacionales (Berdoulay, 1982) A partir de los años 50s hasta los 70s, los estudios regionales desaparecerían paulatinamente, incluso en Francia misma, quedando circunscritos al alero de algunos centros de investigación, focalizados en los estudios de áreas regionales y muy vinculados con las políticas de antiguos imperios coloniales, de algunas de las potencias europeas (Gottmann, 1949).

\subsection{La geografía tropical de Burdeos}

El caso de Burdeos sirve como ejemplo para ilustrar la transformación de la geografía regional en paralelo a los estudios coloniales y tropicales (Gourou y Rivet, 1948; Gourou, 1982). Lejos de la idea común que tiende a explicar que la geografía regional se desvanece a partir de los años 50s, nos encontramos con un interesante proceso de reconversión o adaptación al nuevo contexto. Es importante mencionar esa etapa para poder entender el resurgir de algunos de los principios regionales a partir de los años 90 s, con el auge del ordenamiento del territorio e incluso de los estudios turísticos.

Por su comercio con ultramar, industrias, inversiones o incluso el Instituto colonial entre otros, Burdeos se posicionó como una de las principales ciudades coloniales, incluso tal vez como "la gran ciudad colonial en Francia" (excluyendo París). Sirvió como modelo para muchas otras ciudades de provincia: como Rochefort que pidió asesoramiento para la organización de su Exposición de 1883, o incluso Niza, para la de 1884, o aún una delegación de Rouen, declaró que tras una visita a la Exposición de 1895, Burdeos había superado todas las ediciones anteriores realizadas en Francia [...] (Lozère, 2005, p.37). 
En el contexto descrito, el Centro de Estudios Coloniales de Burdeos permitió desarrollar y difundir muchos de los conocimientos regionales más allá de los años 30s. Con el cambio paulatino político e ideológico asociado a la descolonialización (Maldonado-Torres, 2008), muchos centros de estudios regionales de corte colonial fueron desapareciendo o, como en el caso de Burdeos, se especializaron en los estudios de áreas tropicales hacia los años 50s. El Institut d'outre Mer de Bordeaux d'Études Régionales de la Universidad de Burdeos tiene una tradición que parte de larga fecha. La tradición de la ciudad de Burdeos junto con Marsella, como uno de los principales puertos hasta inicios del siglo XX, que unía la metrópoli con su variado Imperio colonial propició el auge en el siglo XIX de una serie de organismos estatales de investigación y de estudios coloniales que se verían obligados a reconvertirse ulteriormente, después de la Primera y Segunda Guerra Mundial, con la pérdida paulatina de territorios de ultramar (Figura 1).

Figura 1. Muelles de Burdeos durante la Primera Guerra Mundial

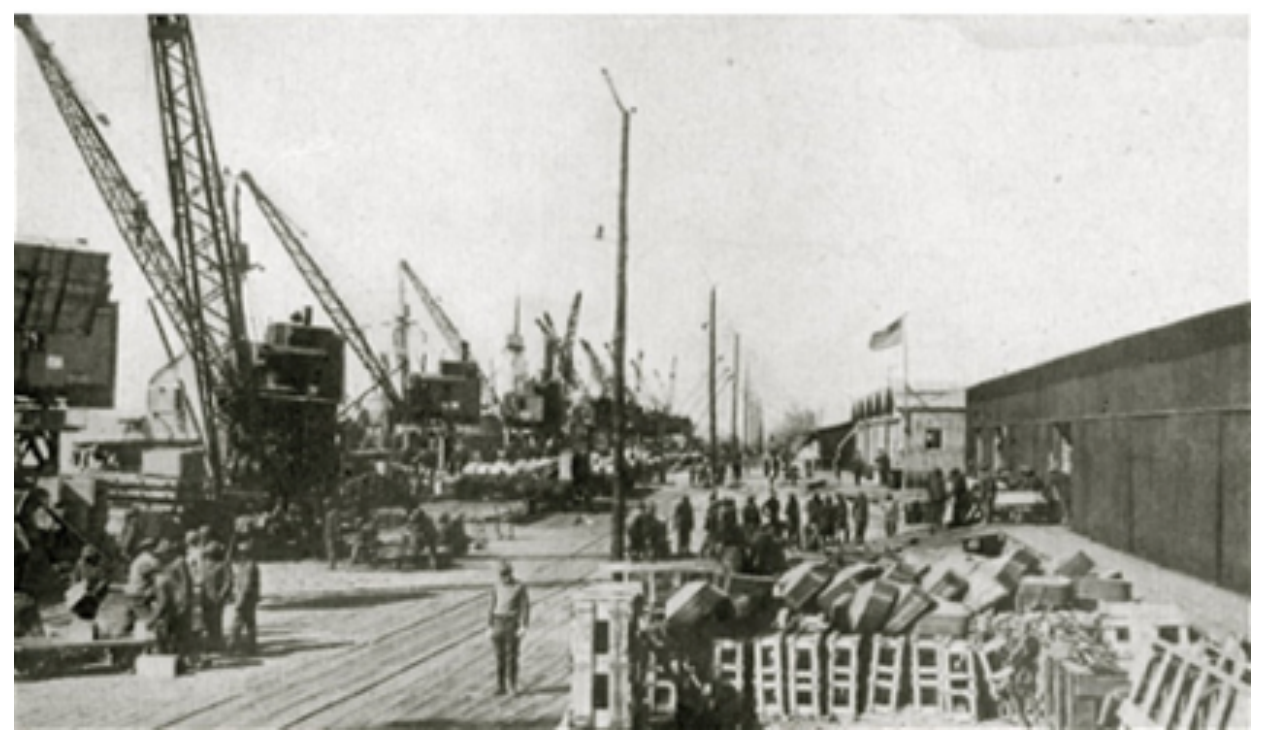

Fuente: Lozère (2005).

La geografía regional no sólo jugaría un papel reconocido en la construcción de los Estados modernos por medio de la educación (Berdoulay, 1982), sino que también desempeñaría un rol importante más desconocido en la reinterpretación de los modelos coloniales hacia una visión más científica y hacia una nueva lógica de relaciones postcoloniales. En cierto modo, también se puede considerar que el proceso de reconversión del modelo colonial ha servido para relanzar la geografía regional dentro de las complejidades multiculturales de la postmodernidad. No sólo hubo rupturas, sino también discursos de continuidad planteados desde la reinterpretación. Los estudios coloniales se reinventarían como nuevos estudios tropicales aportando una cierta continuidad para muchos geógrafos regionales. Bajo los trópicos el regionalismo encontró un refugio geográfico y temático, a pesar de ser ignorado desde la metrópoli. 
La geografía en Burdeos prosperó entre las corologías tradicionales de la geografía aún decimonónica, asociadas a las colonias y al auge de la visión regionalista, fuertemente anclada en una tradición local y de carácter rural. El resultado, fue un fuerte Departamento de Geografía anclado en esa doble faceta universal y local que dejaría una profunda influencia en la región, en Francia y a escala internacional. La transformación de la revista de los Cahiers d'outre-mer: revue de géographie de Bordeaux a la Revue de géographie de Bordeaux, evidenciada a partir del homenaje realizado por Alain Huetz De Lemps a Guy Lasserre es un excelente testimonio que ilustra el auge y transformación del Departamento de Geografía de Burdeos que supo adaptarse al proceso de descolonización y llegar hasta la actualidad, centrándose más en la vertiente regional local.

Cuando Louis Papy y Eugène Revert fundaron nuestra revista, en 1948, vieron en Guy Lasserre, a un joven y entusiasta geógrafo egresado que realizó en el primer número un profundo estado del arte sobre el Nordeste de Brasil. En 1957, Louis Papy decidió reforzar el equipo de Cahiers, con la incorporación de Guy Lasserre como secretario. En 1962, se convertiría en el codirector junto con el Decano Louis Papy y Henri Enjalbert. Durante cerca 30 años, va a ejercer el cargo con aplomo y esmero. Los Cahiers d'Outre-Mer deben mucho a Guy Lasserre y están estrechamente vinculados con su vida [...] En esa época la Universidad reanudó sus relaciones con las Antillas, pese a la partida de Pierre Gourou, nombrado profesor en el Collège de France. Louis Papy reorganizó la Sociedad de Geografía y relanzó junto con la Cámara de Comercio, el Institut Colonial (rebautizado en 1947 como Institut de la France d'Outre-Mer), para ampliar el campo de acción de los geógrafos de Burdeos. El nombramiento de Eugène Revert, quien había pasado varios años en Martinica, permitió a Louis Papy ir concretando el deseo de orientar la geografía de Burdeos hacia países lejanos así como fundar los Cahiers d'OutreMer [...] En 1965, el Decano Papy pide al CNRS la creación de un centro especializado en el estudio de los países tropicales, con el aval de Pierre Gourou y de Pierre Monbeig. Este nuevo laboratorio tomará el nombre propio de CEGET (Centro de Estudios de Geografía Tropical). El edificio es construido en el nuevo campus universitario y Guy Lasserre dirigirá todo el proceso [...] y se convertirá en su primer director, en 1967, delegando la vicedirección a Pierre Vennetier, quien había defendido su tesis sobre Pointe-Noire y era profesor instructor [...] En 1970, obtuvo del Ministerio de los DOM-TOM [Territorios Ultramar] la creación de un Centre National de Documentation des Départements d'Outre-Mer (CENADDOM), que fue instalado en el CEGET. Este organismo fue codirigido por Guy Lasserre y Jacques Menauge, aportando mucha ayuda a los servicios de los funcionarios y universitarios interesados en los asuntos de Ultramar. En la Universidad, se apoyó la creación del Centre d'Etudes des Espaces Tropicaux (CRET), dirigido por Christian Huetz de Lemps. A petición de Fernand Braudel, desempañará un papel esencial en la creación de la Maison des Sciences de l'Homme d'Aquitaine, que será dirigido por su colega y amigo Jean Borde. Fue igualmente uno de los cofundadores de la Ecole Internationale de Bordeaux. Guy Lasserre era solicitado por todas partes y estuvo siempre presente para escuchar y ayudar a sus amigos dispersos por todo el mundo [...] Redactó igualmente capítulos en manuales escolares [...] y manuales sobre las Antillas francesas [...] (Huetz de Lemps, 2001, p.345). 
Las necesidades específicas de especialización en medios de latitudes intertropicales produjeron el interés por mantener una visión particularista sobre unos espacios particulares y complejos (Robic, 1992). Estas necesidades fueron especialmente importantes en medios higienistas y médicos, que buscaban antídotos y vacunas a las numerosas enfermedades específicas desarrolladas en esas latitudes, pero se extendieron también a los estudios de corte más humano, histórico y antropológico, que pudieron seguir prosperando, a pesar de haber quedado desfasados en el ambiente científico del momento. En ese contexto, la geografía regional y su visión holística encontraría un refugio, aunque fuertemente criticada desde las perspectivas ideológicas del momento.

Gracias a centros como el de Burdeos, que supieron valorar el legado tropical al margen de lo político, pudieron sobrevivir algunos de los conocimientos de esa perspectiva geográfica y convertirse en la actualidad en un elemento esencial para la renovación de la disciplina desde la revisión crítica postmoderna. Antiguos profesores eméritos pudieron seguir impartiendo algunos cursos en pre y postgrado, transmitiendo esa visión diversa de la geografía, respecto de las tendencias neopositivistas y radicales imperantes en el momento.

A la sombra de los trópicos los geógrafos regionales encontraron un refugio. La riqueza no sólo natural, sino histórica y cultural, en los trópicos no sólo abriría la visión de la geografía, ante las nuevas dudas epistemológicas de la postmodernidad de finales del siglo XX, frente a la caída de dogmas e ideologías fijas, sino que a su vez influenciaría también al renacer de investigaciones en campos variados de las humanidades, como en los estudios de la literatura anglohablante del Caribe, por ejemplo. El trópico supone una verdadera revolución en relación con las humanidades y sacude todos los preceptos acuñados, desde la complejidad de la particularidad y los mestizajes, generando contradicciones y controversias difíciles de entender desde las lógicas unívocas de una academia con prejuicios previos, tanto liberales como marxistas.

En la actualidad, las diferencias y márgenes empiezan a reconsiderar el objeto de estudio y permiten replantear a su vez la revisión misma de todos los conceptos, en un proceso de relativización. La norma se empieza a ver como una imposición y la experimentación empírica de la visión ideográfica como una liberación desde la cual poder revisar y comprender nuestras sociedades y espacios, en concordancia con los conocimientos exoacadémicos. Lo vernáculo vuelve a ser reconsiderado y considerado como forma de resistencia frente a lógicas racionales homogeneizadores. En las particularidades muchas sociedades encontrarán refugio, entrando en lógicas conservacionistas, incluso desde planteamientos tradicionalmente liberales, como en el caso medioambientalista.

\section{La revisión regional contemporánea}

La revisión contemporánea del legado regional dentro del mundo académico ha permitido resituar la importancia de temas como el referente, el sujeto y la experiencia dentro de la ciencia, que son herederos del legado regional. No obstante, la academia sigue teniendo más dificultad para poder revisar el legado histórico, al margen del colonialismo, en temas como, por ejemplo, el rol de esos centros en la difusión de la 
geografía a nivel mundial, o en la formación de especialistas internacionales que están en el origen de numerosos departamentos de geografía por el mundo, o en el rico legado documental geográfico (fotos, mapas, trabajos de campo, literatura) que sigue silenciado al no poderse desvincular del colonialismo.

La perspectiva postcolonial construye una dialéctica constructiva que permite incorporar la revisión de ese rico legado histórico de la disciplina para incidir a su vez, en una mejor comprensión de la complejidad y derivación del colonialismo

\subsection{El rescate de la experiencia}

La comprensión de los territorios se establece en la mirada regional, desde un exhaustivo conocimiento de la realidad concreta sobre el trabajo de campo. El aprendizaje de la visión holística característica de la geografía es un largo proceso desde la mirada que se desarrolla justamente a partir de la experimentación en los trabajos de campo (Figura 2).

Figura 2. Trabajo de campo en los Pirineos, en cursos postgrado de Burdeos (años 60s)

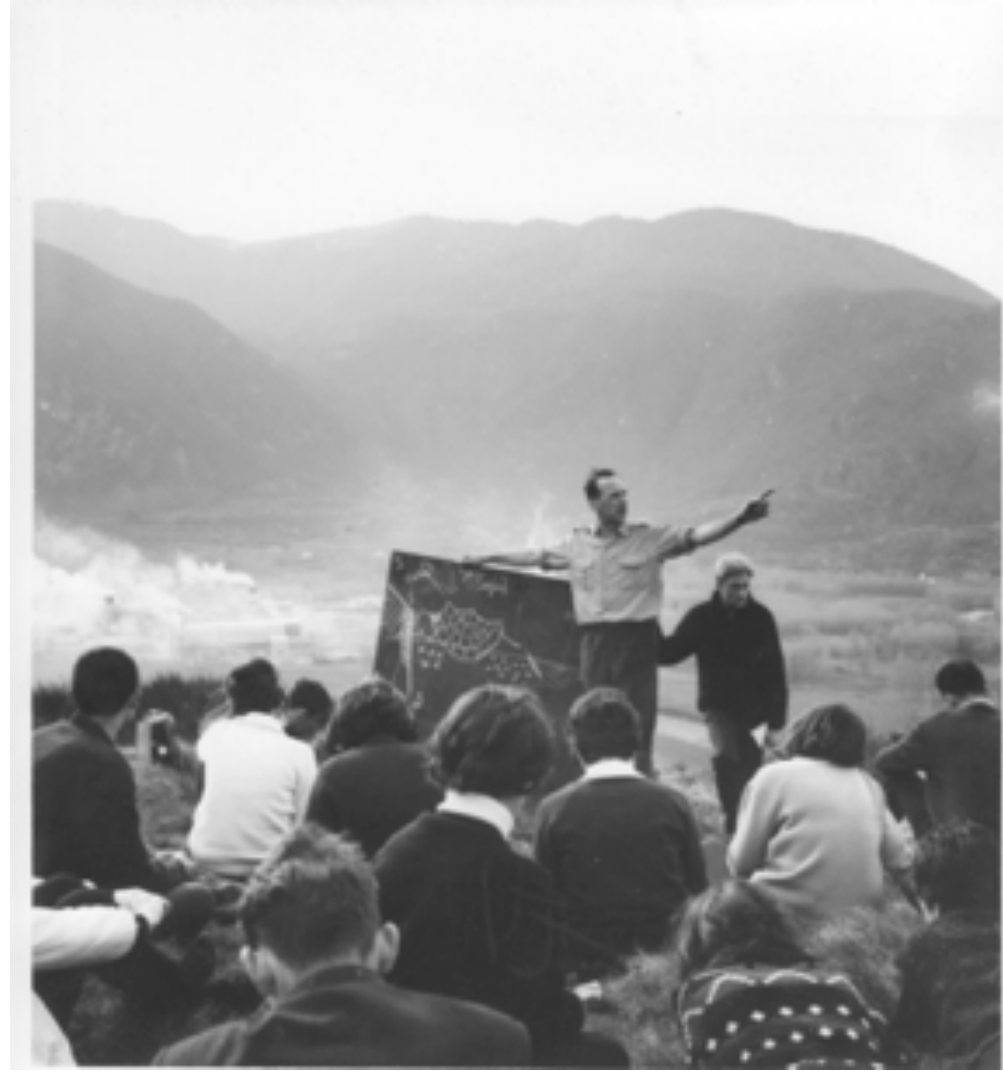

Fuente: Archivo particular cedido por P. Ilabaca.

El trabajo de campo para la geografía regional representa la piedra angular de su enseñanza. Por un lado, es justamente la experiencia del trabajo de campo la que 
permite desarrollar ciertas particularidades del geógrafo, como son, en primer lugar, la capacidad de observación de los elementos de todo tipo del territorio (tanto naturales como antrópicos), en segundo lugar, el establecimiento de interrelaciones entre los distintos elementos en una visión temporal y espacial razonada y, en tercer y último lugar, el aprendizaje de la síntesis (mirada) a partir de la capacidad de saber explicar de forma simple las interrelaciones observadas en el territorio. Duramente cuestionado por sus detractores por simplista, la mirada regional es justamente plural y compleja.

Por otro lado, la realización del trabajo de campo en geografía representa una declaración de principios, respecto, en primer lugar, del planteamiento ideográfico regional. El aprendizaje de las capacidades inductivas desde la observación empírica se utiliza para la comprensión concreta y particular de cada lugar. En segundo lugar, el contacto directo con el espacio permite establecer un vínculo participante, en el cual el observador es sujeto y parte de ese mismo todo. En ese sentido, en el trabajo de campo se aprende a manejar el rol del geógrafo respecto de la subjetividad, así como de su implicación social como parte de ese todo.

En la escuela regional los trabajos de campo eran parte integrante de los estudios, como muy bien lo indican muchos de los laboratorios regionalistas que prosperaron en tiempos, e incluso en su derivación hacia centros de investigación estrechamente vinculados con territorios coloniales y ulteriormente centrados en áreas como los trópicos. El caso de la Universidad de Burdeos no fue la excepción, y son numerosos los testimonios de los egresados - a partir de las entrevistas realizadas - que recuerdan con mucho cariño las salidas locales, en la región de las Landas, así como el aprendizaje a partir de las anotaciones en los carnets de notas. Más allá del aprendizaje, el trabajo de campo también representa una vivencia entrañable para el estudiante en su contacto con el grupo y la comunidad de estudio. El carácter local de los trabajos de campo, permite una interacción directa con el habitante, estableciendo una profunda vinculación con el conocimiento geográfico en un sentido transversal, sin distinción entre academia y vernáculo. Esa mirada de estudio académica, de igual a igual, se acerca mucho más a los postulados postcoloniales y está mucho más lejos paradójicamente de los postulados coloniales a los que estaban sujetos.

El trabajo de campo convierte a la disciplina geográfica en algo interesante y a la vez entretenido, con un carácter inclusivo y de amplia difusión social. Frecuentemente desde el ámbito académico geográfico se olvida que somos todos constructores de territorialidades. El geógrafo regional en ese sentido ha desempeñado un rol esencial como observador, transmisor y difusor pedagógico de esas territorialidades. El homenaje a Louis Papy en la revista Norois, realizado por Paul Fénelon, es un buen ejemplo:

Para recordar mejor quien fue este eminente geógrafo, ese espíritu brillante y generoso, deben haberlo acompañado por las colinas del Armagnac, haber subido las montañas del Adour y del País Vasco o haber escuchado sus ricas conversaciones trufadas de ideas nuevas, sin faltar a un toque de humor casi británico. Nos ceñiremos a mencionar su alta y esbelta silueta sobre los promontorios del Aunis y de Saintonge, dos provincias a las cuales dedicó una bella obra ilustrada en portada con una acuarela dibujada por su madre a la cual siempre veneró. Nos acordaremos de sus preciados consejos y de toda la ayuda que nos dedicó durante cerca de medio siglo, que atestiguan las más de 500 cartas clasificadas en mis archivos. Entre 
sus obras, destacaron los Cahiers d'Outre-Mer, revista que posicionó la Universidad de Bordeaux, en la vanguardia de las investigaciones geográficas sobre África y América, y su monumental Géographie régionale de la France en 16 volúmenes, que dirigió prodigiosamente Charentes, sobre Burdeos o sus queridas Landas. Resaltamos sobre todo su bondad y generosidad en la dedicatoria en su tesis a sus dos hermanos (Raymond, el agrónomo, y Roger, el politécnico) desaparecidos cuando eran aún jóvenes, el excelente jefe de familia que era, así como su contribución sin titubeos a la lucha contra el ocupante después del desastre de 1940, sin descuidar al brillante y comprensivo profesor para miles de estudiantes (Fénelon 1990, p.77).

En el caso de Burdeos, el medio regional de Aquitania (Papy, 1941, 1982; Enjaelbert, 1953, 1967; Fénelon, 1951) y de los Pirineos (Cavaillès, 1931) sirvieron como escenografía perfecta para ilustrar con trabajos de campo y ejemplos, todos los conceptos teorizados de la geografía regional, sin por ello contradecir su vocación universal. La difusión pedagógica de su legado se propagó gracias a sus discípulos (profesores originarios del lugar pero formados en Burdeos y que regresaban a sus países) en áreas de estudio lejanas e incluso están en el origen de nuevos departamentos de geografía, sin renegar de su propia identidad. La revisión actual de ese hecho demuestra la validez de los planteamientos académicos basados en un profundo respeto y admiración por la diversidad de referentes y sin ninguna intención de imposición académica o colonial.

La capacidad también de conciliar la visión universal sobre el trópico, sin desmedro por la identidad local aquitana puede entenderse en la actualidad como una característica de los postulados geográficos más contemporáneos. El presente análisis detallado del trabajo de campo en el caso de la Universidad de Burdeos es un buen ejemplo para demostrar cómo se puede establecer una revisión que, si bien no pierde de vista el contexto histórico en este caso vinculado con el modelo colonial, puede a su vez recuperar una rica experiencia académica, en este caso concreto centrada en el rol que desempeña el trabajo de campo para la geografía regional. El análisis del trabajo de campo permite poner a la luz el discurso de una visión particular y respetuosa ante la diversidad de referentes, que dista de los postulados políticos supremacistas del colonialismo y, por el contrario, apoya curiosamente algunos de los antecesores de los postulados postmodernos (la consideración del sujeto) y postcoloniales (la consideración del otro) dentro del mundo académico (Loomba, 2015).

El tropicalismo encierra la comprensión de la diversidad enriquecedora del otro, al margen de prejuicios. Este ejemplo sirve para mostrar la necesidad de reconsideración del legado colonial como la mejor garantía para deconstruir los discursos supremacistas y evitar su repetición bajo nuevas formas. La negación y olvido no demuestran la superación sino el temor a su reconocimiento.

\subsection{La revalorización del discurso}

Uno de los ámbitos más importantes dentro de la visión regionalista es el arte de saber explicar y narrar (Berdoulay, 1982), tanto oralmente como por escrito - $\mathrm{e}$ incluso hoy podría ampliarse a otros medios de comunicación visuales o cibernéti$\cos$ - L La relación de la geografía con la escritura y la pedagogía son tan estrechas para la formación del geógrafo como lo pueden ser para la del periodista. La mayoría 
de los autores regionalistas se caracterizaron por ser excelentes oradores y escritores, lo que acentúa una vez más el carácter interdisciplinario y humanista en su formación, sin descuidar el manejo del lenguaje cartográfico, así como un especial apego por el manejo de varios idiomas, dada su experiencia internacional en sus cátedras en diversos países y viajes. Muchos profesores de la escuela de Berkeley (como Sauer o Wagner, por ejemplo) dominaban ocho lenguas. La prosa de ciertos escritores (como Pla o Verdaguer, en el contexto catalán) atestiguan la estrecha relación entre la geografía y la literatura (Tort i Donada, 2007). Este caso no es excepción y podemos encontrar estrechos vínculos en otros ámbitos: desde la literatura romántica alemana europea (Herder) o la literatura fantástica latinoamericana (Rulfo, Borges o Sepúlveda) hasta ulteriormente algunos de los géneros cinematográficos como el neorrealismo italiano (Rosselini, Visconti), por no mencionar las corrientes pictóricas, desde el impresionismo hasta el hiperrealismo. La vinculación de la geografía con las artes recién empieza a reconocerse a pesar de haberse dado sin problemas durante el auge de la geografía regional, de corte ideográfico.

No podemos dejar de mencionar igualmente, la estrecha relación de los geógrafos regionales con el desarrollo del conocimiento histórico. Las influencias de las corrientes históricas de la Escuela de los Annales, estructuralistas y postestructuralistas, incidirán brillantemente en la geografía de corte historicista o en la historia de corte geográfico, según se mire, de autores como Vicens Vives, Vilar o Braudel.

\section{Conclusión: la mirada tropical}

La mirada que aportó la geografía sobre las regiones tropicales representa un rico legado para la disciplina que se encuentra todavía parcialmente en revisión. Por una parte, dentro de la disciplina de la geografía académica se ha rescatado recientemente el valor de ciertos aspectos, en relación al papel del referente y del sujeto así como del relato como forma de lenguaje para transmitir un conocimiento geográfico (Berdoulay, 2000; Berdoulay, Laplace-Treyture y Arnauld de Sartre 2010). La perspectiva de la geografía postmoderna ha permitido reconocer el lugar del conocimiento ideográfico, así como la importancia de la representación y de sus construcciones, que aportó la geografía regional. La descripción geográfica, lejos de una mera enumeración puede representar una interpretación de los lugares, individualmente, desde los espacios vividos (percepción), pero también colectivamente, como imagen social. La revisión regional ha permitido incorporar un legado que ha puesto a la geografía dentro de los debates contemporáneos con mayor facilidad que otras disciplinas, al haber ya considerado aspectos que desde la ortodoxia científica clásica, eran ajenos.

Por otra parte, en relación al contexto político del legado geográfico muy ligado al colonialismo, el debate sigue aún en el aire, con cierta dificultad para poder ser abordado desde las posturas postcoloniales más ortodoxas (Young, 2016). No obstante, la revisión, como hemos visto con el caso del presente estudio de la geografía tropical de Burdeos, permite, por el contrario, aportar elementos importantes al debate postcolonial contemporáneo. La mirada regional particular centrada en el respeto a la diversidad y pluralidad de los referentes permite, por un lado, rescatar un legado patrimonial documental regional por ahora olvidado (fotos, libros, películas, etc.) con más de cien años de historia, que puede revelar mucha información para el 
conocimiento de muchos pueblos y lugares, algunos ya desaparecidos (etnografía). También, por otro lado, ese legado aporta una mirada desde el respeto y diversidad de los referentes, muy lejos de los estereotipos del colonialismo con el que se asocia de forma simplista. El criticado carácter excepcional de la geografía regional por parte de la geografía académica reviste una mirada diversa sobre los territorios, lejos de modelizaciones y estandarizaciones. El tropicalismo reivindica una diversidad al margen de las normas culturales homogeneizadoras.

\subsection{La revisión del referente}

Las escuelas geográficas alemana y francesa usaron las particularidades, siguiendo el modelo tradicional de las visiones vernáculas (Santini, 2017, 2018a), para el proceso de materialización de sus idearios nacionales basados en lo particular y en lo universal, respectivamente. Ambas escuelas tuvieron un profundo impacto igualmente en la geografía de Estados Unidos, con el desarrollo, por un lado, de la geografía cultural de la Escuela de Berkeley (Sauer, 1925), centrada en el estudio de los paisajes (Butzer, 1982) y muy influenciada por la herencia alemana y, por otro lado, en el auge del regionalismo americano (Odum, 1938), muy influenciado por la geografia regional francesa (Vallaux, 1911; Meinig, 1979).

Ante la crisis de las estructuras racionales y de mercado contemporáneas, el pensamiento occidental ha encontrado un refugio en lo local frente al escenario de multiculturalidad creciente (Castells, 1997). Desde los estudios postmodernos, la geografía ha replanteado el rol de las representaciones colectivas, pero sólo como objeto de estudio y no como parte y artífice de ellas (Berdoulay, 1988). La negación de la aceptación del sujeto, por una cuestión epistemológica, ha impedido el restablecimiento de la estrecha dialéctica con la sociedad. Se habla del sujeto, se observa el sujeto, pero no se es parte de él. No sólo la falta de compromiso (corriente radical) sino el rol como sujeto (behaviorismo), fueron ya denunciados en los 70 s, pero estas críticas eran difícilmente asumibles desde una postura académica nomotética. Es solo con el asentamiento más reciente de los postulados estructuralistas (Braudel, 1986), el ulterior desarrollo de la fenomenología (Bourdieu, 2003) y el reencuentro con la tradición culturalista en los 90s (Wagner, 1972) y del renovado interés por los paisajes (Wagner y Mikesell, 1962; Gade, 1967, 1999; Gourou, 1984) que se empieza a reconsiderar todo ese legado (Price y Lewis, 1993; Foote, 1994; Murphy, Jordan-Bychkov y Jordan, 2002; Claval, 2003).

La gran fortaleza del conocimiento regional, se basó justamente en encontrar en la interpretación de lo particular, genres de vie (Vidal de La Blache, 1911), un punto de discusión con el resto de la sociedad. Todos participaban desde lo vernáculo y lo académico en la construcción de una comprensión común de las formas o artes de vida compartida (Vidal de La Blache, 1913). Por el contrario, en la visión social culturalista postmoderna (Nash, 2002) sigue existiendo ese sesgo académico de imposición de una verdad especializada sobre el resto, al no asumir la particularidad (Castells, 1997). El gran éxito de la geografía regional y del conocimiento ideográfico es que parte de la experimentación, entendida como empirismo científico. De esta manera, el compartir y participar de una misma vivencia fue acercando el conocimiento geográfico a todos.

La formación plural de los profesores de esa escuela, junto con su vocación pedagógica y de difusión, sin descuidar su profundo compromiso con la sociedad, 
constituyen perfiles académicos de carácter humanista muy acordes con la visión integradora de la geografía francesa (Brunhes, 1920). El caso del profesor Pierre Camena d'Almeida de la escuela de Burdeos, permite ilustrar todas estas cualidades, como aparece reflejado en un homenaje realizado a su persona por uno de sus discípulos Henri Cavaillès:

Egresado de la École Normale en 1883, junto con Joseph Bédier, Stéphane Gsell y Emile Male, fue, como Lucien Gallois, uno de los más acérrimos discípulos de Vidal de La Blache. En 1886-1887 estará en Alemania (Marburg, Leipzig y Berlin), donde recibe clases de Ratzel y de Richthofen. En 1893 obtiene su grado de doctor por una tesis sobre Los Pirineos: conocimiento geográfico de la cordillera y una tesis complementaria en latín sobre El Mar Caspio según los antiguos, en una visión histórica. En 1889 es nombrado profesor interino y asociado en Caen y en 1899 pasa definitivamente a ser profesor en Burdeos hasta su jubilación en 1935 [...] Su docencia se ve complementada por la publicación de manuales para la enseñanza secundaria, conjuntamente con Vidal De La Blache, $[\ldots]$ y una nutrida producción científica, complementada por su rica formación y manejo del alemán, inglés, ruso e incluso polaco, adquiridos durante sus viajes a Alemania (18861887), Rusia (en 1894 hasta el lago Baikal) y Turkestan (en 1910). Estuvo atraído por los fenómenos naturales y en particular sobre sus efectos sobre la población, así como por sus adaptaciones y reacciones frente al medio [...] pero igualmente por temas históricos $\mathrm{y}$, en especial, temas militares, como atestiguan sus estudios sobre el ejército ruso (1896) y el ejército alemán antes y después de 1914-1918 (1919) (Cavaillès, 1945, p.67)

$\mathrm{Su}$ formación ilustra la estrecha interrelación de la geografía alemana y francesa, así como el carácter humanista de la misma (Santini, 2018b), así como un amplio dominio lingüítico. Su visión empírica del conocimiento se ve reforzada con sus numerosos trabajos de campo a escala mundial, así como, por derivación, con la amplia influencia de sus conocimientos, no sólo en los discípulos dejados en Burdeos, sino también en todos los lugares visitados. Esta visión rompe categóricamente con la idea ulterior de una geografía científica nomotética y especializada, así como con la asociación simplista con el colonialismo, que sirvió más como una excusa para poderla dejar de lado.

El comprender la importancia de la correlación de un territorio con una comunidad, a partir de la construcción de sus referentes territoriales colectivos, es uno de los grandes aportes de la geografía regional francesa, bien lejos de los intereses de justificación política supremacista por los que fueron simplificados y criticados. La perspectiva contemporánea postcolonial (Jazeel y McFarlane, 2010) permite discernir el legado del tropicalismo, en su diversidad, de la carga de los referentes históricos coloniales. La construcción del referente tropical además no fue sólo el fruto de la mirada occidental académica sobre el otro sino que también permitió divulgar unas realidades que eran ignoradas hasta la fecha. Los orígenes de la pluralidad conceptual y de referentes surgen, paradójicamente, de parte del legado que también sirvió para respaldar el modelo colonial. La diferencia subyace entonces no tanto en el legado, sino más bien en la interpretación e intencionalidad política que reviste. Ese punto, más allá del caso concreto de Burdeos, permite entender la importancia de divulgar ese legado como una forma de aprendizaje y tolerancia - o no temor- hacia la 
diversidad y, a la vez, como antecedente histórico para concienciar de los peligros de la fácil manipulación política a partir de planteamientos binarios de corte populista.

\section{2. ¿Los trópicos descoloniales?}

La existencia misma del concepto de "trópico" puede ser entendida tanto como una forma de imposición cultural sobre la realidad de territorios propios, como también una mirada académica para comprender la diversidad del otro. Los preceptos postcoloniales más estrictos apuntan a una visión más ortodoxa excluyente (crítica a un eurocentrismo científico), aunque ambas visiones, desde sus propias contradicciones, son parte de un mismo todo. No podemos desligar el interés imperialista y colonialista que permitió financiar las expediciones y centros de investigación regional de los imperios europeos, pero, a su vez, también es absurdo negar el rico legado plural existente en la actualidad. Así, por ejemplo, muchos territorios desaparecidos o en vías de desaparición pueden en la actualidad encontrar en los legados etnográficos y geográficos de testimonios propios, elementos muy útiles para su proceso de reivindicación.

Desde esa perspectiva inclusiva, los trópicos encuentran su lugar dentro de los estudios postcoloniales, convirtiéndose en unos "trópicos post-coloniales" o bien "post-tropicales". Las cartografías y expediciones coloniales son el testigo de una imposición que debe ser denunciada, pero aportan a su vez un legado de conocimiento que las mismas culturas implicadas merecen poder disponer para la restitución de sus referentes. El interés de los geógrafos regionales franceses por los genres de vie sirvió para la justificación de las representaciones nacionales (Nadal, 2007), pero también arrojaron luz sobre mundos diversos, basados en adaptaciones diversas al medio natural que pueden también ser de interés para todos, ante los retos demográficos, ambientales y ecológicos globales actuales.

Los trópicos existen de la misma manera que los centros de investigaciones antárticas - que no plantean los mismos problemas éticos, en este caso por haber permanecido deshabitados - o árticas, con independencia de las connotaciones políticas implícitas que siempre estuvieron estrechamente relacionadas con la geografía. No olvidemos que la misma Geografía de Estrabón, considerado el primer testimonio de la disciplina, nos permite entender la visión del Ecúmene (la representación del mundo) de cerca de dos mil ochocientos años - al incluir las alusiones al mundo homérico-, al margen de la vinculación esclavista familiar o de la imposición política para el establecimiento del Imperio romano. Si bien no podemos olvidar el contexto tampoco podemos borrarlo.

\section{Agradecimientos}

A Joan Vilà i Valentí, in memoriam. Su legado está presente en este artículo. 


\section{Referencias}

Bailón, R. O. F. (2015). Propuestas epistémicas descoloniales en Sakubel k’inal jajchwinikLa Aurora Lacandona y Canek. Revista Iberoamericana de Ciencias, 2(1), 91-102.

Balandier, G. (1973). Teoría de la descolonización. Buenos Aires: Editorial Tiempo Contemporáneo.

Berdoulay, V. (1981). La formation de l'école française de géographie: 1870-1914 (Vol. 11). Paris: Bibliothèque nationale.

Berdoulay, V. (1982). La métaphore organiciste. Annales de géographie, 91(507), 573-586.

Berdoulay, V. (1988). Des Mots et des Lieux: la dynamique du discours géographique. Pau: Editions du Centre national de la recherche scientifique.

Berdoulay, V. (2000). Le retour du refoulé. Les avatars modernes du récit géographique. En J. Levy y M. Lussault (Dirs.), Logiques de l'espace, esprit des lieux. Géographies à Cerisy (pp.111-126). Paris: Belin.

Berdoulay, V., Laplace-Treyture, D., y Arnauld de Sartre, X. (2010). La question du sujet et la géographie. Cahiers de géographie du Québec, 54(153), 397-418.

Borde, J. (1966). Les Andes de Santiago et leur avant-pays: étude de géomorphologie. Paris: Union Française d'Impression.

Borde, J. (1980). Le Chili: la terre et les hommes. Paris: Éditions du Centre National de la Recherche Scientifique.

Bourdieu, P. (2003). El oficio de cientifico. Ciencia de la ciencia y reflexividad. Barcelona: Anagrama.

Braudel, F. (1976). El Mediterráneo y el mundo mediterráneo en la época de Felipe II (Vol. 2). México: Fondo de cultura económica.

Braudel, F. (1986). La historia y las ciencias sociales. Barcelona: Alianza editorial.

Brunhes, J. (1920). La géographie humaine. Paris: Félix Alcan.

Butzer, K. W. (1982). Archaeology as human ecology: method and theory for a contextual approach. Cambridge: Cambridge University Press.

Capitanelli, R., y Zamorano, M. (1972). Geografía regional de la Provincia de San Luis. Boletín de estudios geográficos, 19(74-77), 1-320.

Castells, M. (1997). Local y global. La gestión de las ciudades en la era de la información. Madrid: Taurus.

Cavaillès, H. (1931). La vie pastorale et agricole dans les Pyrénées des Gaves, de l'Adour et des Nestes: étude de géographie humaine. Paris: A. Colin.

Cavaillès, H. (1945). Pierre Camena d’ Almeida (1865-1943). Annales de Géographie, 54(293), 67-68.

Claval, P. (2003). La géographie du XXI siècle. Paris: L'Harmattan.

Claval, P. y Sanguin, L. (Eds.). (1996) La géographie française à l'époque classique (19181968). Paris: L'Harmattan.

Edmondson, B. (1999). Caribbean romances: The politics of regional representation. Chalottesville, VA: University of Virginia Press.

Enjaelbert, H. (1953). Comment naissent les grands crus: bordeaux, porto, cognac: Première partie. Annales. Histoire, Sciences Sociales, 8(3), 315-328.

Enjaelbert, H. (1967). Les montagnes calcaires du Mexique et du Guatemala. Annales de Géographie, 76(413), 29-59.

Estermann, J. (2014). Colonialidad, descolonización e interculturalidad. Apuntes desde la Filosofía Intercultural. Polis, Revista Latinoamericana, 13(38), 347-368. 
Fénelon, P. (1951). Le Périgord, étude morphologique. L’information géographique, 15(2), 77-80.

Fénelon, P (1990). Louis Papy. Norois, 37(146), 105.

Foote, K. (1994). Re-Reading Cultural Geography. Austin: University of Texas Press.

Gade, D. W. (1967). The guinea pig in Andean folk culture. Geographical Review, 57(3), 213-224.

Gade, D. W. (1999). Nature and Culture in the Andes. Madison, WI: University of Wisconsin Press.

Gallois, L. (1909). Régions naturelles et noms de pays: étude sur la région parisienne. Paris: Armand Colin.

González, M. C. (2003). An ethics for postcolonial ethnography. En R. P. Clair (Ed.), Expressions of Ethnography: Novel Approaches to Qualitative Methods (pp.77-86). Albany, NY: State University of New York Press.

Gottmann, J. (1949). Mer et terre: Esquisse de géographie politique. Annales. Histoire, Sciences Sociales, 4(1), 10-22.

Gourou, P. (1965). Les paysans du delta tonkinois: étude de géographie humaine. Tome 1. Paris: Mouton.

Gourou, P. (1982). Terres de bonne espérance: le monde tropical. Paris: Plon.

Gourou, P. (1984). Riz et civilisation. Paris: Fayard.

Gourou, P., y Rivet, P. (1948). Les pays tropicaux: principes d'une géographie humaine et économique. Tome 3. Paris: Presses Universitaires de France.

Grosfoguel, R. (2006). La descolonización de la economía política y los estudios postcoloniales: transmodernidad, pensamiento fronterizo y colonialidad global. Tabula rasa, (4), 17-46.

Gsell, S. (1997). Atlas archéologique de l'Algérie. Tome 1 ( $\left.2^{\mathrm{a}} \mathrm{ed}\right)$. Alger: Agence Nationale d'archéologie et de protection des sites et monuments historiques.

Huetz de Lemps, A. (2001). Guy Lasserre (1920-2001). Les Cahiers d'Outre-Mer, (216), 345-368.

Ilabaca, P. (1993). El riesgo de anegamiento en la llanura litoral de Concepción - Talcahuano. Proposición metodológica. Revista Geográfica de Chile Terra Australis, (38), 65-72.

Jazeel, T., y McFarlane, C. (2010). The limits of responsibility: a postcolonial politics of academic knowledge production. Transactions of the Institute of British Geographers, 35(1), 109-124.

Kapoor, I. (2008). The postcolonial politics of development. Londres y Nueva York: Routledge.

Laugénie C. (1971). La structure et l'orogénèse des Andes chileno-argentines. Cahiers de géographie du Québec, 15(35), 267-287.

Lindón, A., Hiernaux, D., y Bertrand, G. (2006). Tratado de geografía humana. Barcelona: Anthropos Editorial.

Loomba, A. (2015). Colonialism/postcolonialism. Londres y Nueva York: Routledge.

López Trigal, L. (2017). Comentario. Demangeon y la respuesta de los geógrafos franceses a la Geopolitik. Geopolitica(s), 8(1), 125-131.

Lozère, C (2005). Bordeaux à l'heure coloniale. Revue Le Festin, (56), 36-45.

Luna-Nemecio, J. (2019). Geografía crítica, educación popular y socioformación: triple fundamentación para pensar el desarrollo social sostenible. En L. G. Juárez-Hernández, J. Luna-Nemecio y C. Guzmán (Coords.), Talento humano, investigación y socioformación (pp.443-469). México: CIFE. 
Maldonado-Torres, N. (2008). La descolonización y el giro des-colonial. Tabula rasa, (9), 61-72.

Meinig, D. W. (1979). The interpretation of ordinary landscapes: Geographical essays. Nueva York: Oxford University Press.

Murphy, A. B., Jordan-Bychkov, T. G., y Jordan, B. B. (2002). The European Culture Area: A Systematic Geography. Lanham, MD: Rowman \& Littlefield.

Nadal, F. (2007). Los nacionalismos y la geografía. Geo Crítica: cuadernos críticos de geografía humana, XII(86). Recuperado de http://www.ub.edu/geocrit/geo86.htm

Nash, C. (2002). Cultural geography: postcolonial cultural geographies. Progress in human geography, 26(2), 219-230.

Nash, M. (2005). La doble alteridad en la comunidad imaginada de las mujeres inmigrantes. En M. Nash, R. Tello y N. Benasch (Eds.), Inmigración, género y espacios urbanos: los retos de la diversidad (p. 17-32). Barcelona: Bellaterra.

Odum, H. O. (1938). American Regionalism: A Cultural-Historical Approach to National Integration. Nueva York: Holt.

Papy, L. (1941). L'homme et la mer sur la côte Atlantique de la Loire à la Gironde: étude de géographie humaine. Bordeaux: Éditions Delmas.

Papy, L. (1982). Atlas et géographie du Midi atlantique (Vol. 15). Paris: Flammarion.

Price, M., y Lewis, M. (1993). The reinvention of cultural geography. Annals of the Association of American Geographers, 83(1), 1-17.

Robic, M. C. (1992). Sur la naissance de l'«espace géographique». L'Espace géographique, 21(2), 140-142.

Russo, D. (2004). Émile Mâle (1862-1954): l'invention de l'iconographie historique. Comptes-rendus des séances de l'Académie des Inscriptions et Belles-Lettres, 148(4), 1641-1650.

Santini, C. (2017). At the Origins of Modern Geography. The Oecumene: an Anthropogeographical Pattern. History of European Ideas, 43(6), 560-569.

Santini, C. (2018a). One Sea, One Humanity. Modeling the Man-Sea Relationship in Friedrich Ratzel's Anthropogeographical Project. Journal of Interdisciplinary History of Ideas, 6(12), 4:1-4:24. DOI : 10.13135/2280-8574/2543

Santini, C. (2018b). Can Humanity be Mapped? Adolf Bastian, Friedrich Ratzel and the Cartography of Culture. History of Anthropology Newsletter, (42). Recuperado de https://histanthro.org/notes/can-humanity-be-mapped/.

Sauer, C. O. (1925). The Morphology of Landscape. Geography, 2(2), 19-53.

Sidaway, J. D. (2016). Postcolonial geographies. En D. Richardson et al. (Eds.), International Encyclopedia of Geography: People, the Earth, Environment and Technology: People, the Earth, Environment and Technology, 1-7. Hoboken, NJ: Wiley. DOI: 10.1002/9781118786352.wbieg1100

Talledos Sánchez, E. (2014). La geografía: un saber político. Espiral (Guadalajara), 21(61), 15-49.

Tomasi, J. (1995). Kymlicka, liberalism, and respect for cultural minorities. Ethics, 105(3), 580-603.

Tort i Donada, J. (2007). Cuatro escritores (Verdaguer, Ruyra, Pla y Manent) en la conformación del "canon paisajístico" catalán. Ería: Revista cuatrimestral de geografia, (73), 351-372.

Unwin, T. (1995). El lugar de la geografia. Madrid: Cátedra.

Vallaux, C. (1911). Le sol et l'état. Paris: Octave Doin et fils. 
Vicens Vives, J. (1961). Historia social y económica de España y América (5 vols.). Barcelona: Editorial Vicens-Vives.

Vidal de La Blache, P. (1908). Tableau de la géographie de la France. Paris: Hachette.

Vidal de La Blache, P. (1911). Les genres de vie dans la géographie humaine. Annales de géographie, 20(111), 193-212.

Vidal de La Blache, P. (1913). Des caractères distinctifs de la géographie. Annales de géographie, 22(124), 289-299.

Vidal de La Blache, P. (2008). Principes de géographie humaine. Paris: Editions L'Harmat$\tan$.

Vilà Valentí, J. (1983). Introducción al estudio teórico de la Geografia. Barcelona: Ariel.

Vilar, P (1962). Catalunya dins l'Espanya moderna. Barcelona: Edicions 62.

Wagner, P. L. (1972). Environments and peoples. Englewood Cliffs, NJ: Prentice-Hall.

Wagner, P. L., y Mikesell, M. W. (Eds.). (1962). Readings in cultural geography. Chicago: University of Chicago Press.

Young, R. J. (2016). Postcolonialism: An historical introduction. Hoboken, NJ: John Wiley \& Sons.

Zamorano, M. (1959). El viñedo de Mendoza. Boletín de Estudios Geográficos, 6(23), 4999.

Zusman, P. (2011). La tradición del trabajo de campo en Geografía. Geograficando, 7(7), 1532.

Zusman, P. (2013). La geografía histórica, la imaginación y los imaginarios geográficos. Revista de Geografia Norte Grande, (54), 51-66. 